\title{
An Analysis of the Particle Formation and Growth Process of the Nanocrystalline Diamond (NCD) Particles on Various Interlayers
}

\author{
Dong-Bae Park ${ }^{1}$, Chan-Hyoung Kang ${ }^{2}$, Dong-Hyuk Cha ${ }^{3}$ \\ ${ }^{1,3}$ Precision Analysis \& Development Lab, Cooperative Equipment Center, Korea Polytechnic \\ University, Shihung, Korea \\ ${ }^{2}$ Departments of Advanced Materials Engineering, Korea Polytechnic University, Shihung, Korea
}

Since diamond has good material properties such as high hardness, excellent wear resistance and high thermal conductivity, it is widely used in cutting tools or wear resistant materials. Two major types of industrial diamond are Microcrystalline Diamond (MCD) and Nanocrystalline Diamond (NCD). MCD is easy to make and cheap, but it has some disadvantages such as high surface roughness and high residual stress.

To overcome these difficulties, many researches on NCD have been actively conducted in these days $[1,2]$. According to the research, using an interlayer during the NCD deposition process can block the cobalt dissolution and carbon absorption, and can reduce the residual stress [3, 4].

In this paper, we analyze the particle formation and growth process of the NCD particles on two types of interlayer, tungsten (W) and titanium (Ti), which are known as good materials for interlayer. The procedure of the investigation is as follows: for the Ti interlayer, at first Ti was sputtered on silicon (Si) substrates. The sputtering process had been performed on a DC magnetron sputter until depth of the sputtered interlayer became $1 \mu \mathrm{m}$. Secondary, the sputtered Ti layer was scratched in an ultrasonic bath contains nano size diamond powder. At third, NCD was deposited on the scratched Ti layer at $600^{\circ} \mathrm{C}$ in $2.45 \mathrm{GHz}$ microwave plasma CVD system. For the $\mathrm{W}$ interlayer, the same process was conducted.

Fig. 1 shows the AFM (Atomic Force Microscopy) images of the surfaces of the W interlayers and Ti interlayer. The surface of W has a barley grain-like shape (Fig.1 (a)), while that of Ti a needle-like shape (Fig.1 (b)). The roughness average measurement of interlayer is $\mathrm{W}=3.39 \mathrm{~nm}$, and $\mathrm{Ti}=8.9 \mathrm{~nm}$. Fig.2 shows the SEM (Scanning Electron Microscope) images of NCD layers deposited on the interlayers. At $\mathrm{t}=0.5$ hour, the NCD/W particles (Fig.2 (a)) are generated slower than the NCD/Ti particles (Fig.2(c)), while at $\mathrm{t}=2$ hours the growth of NCD/W particles (Fig.2 (b)) is faster than that of NCD/Ti (Fig.2 (d)) particles. In the case of the W interlayer, NCD particles were coalesced and evolved to a film within 0.5 hour. It is mainly due to the fact that the diffusion of carbon species on the $\mathrm{W}$ interlayer was fast. The slower diffusion of carbon on the Ti interlayer is considered due to be slower film growth than that on the $\mathrm{W}$ interlayer.

To obtain depth data, the two specimens are cut by a FIB (Focused Ion Beam) and the sections are observed by a STEM (Scanning Transmission Electron Microscope) as shown in Fig.3. The figure reveals that the gap of void on the NCD/W (Fig.3 (a)) is narrower than that of the NCD/Ti (Fig.3 (b)), and the thickness of the NCD/W is higher than that of the NCD/Ti.

From the above results it can be concluded that the organizational structure of the NCD/W is better than that of the NCD/Ti. The reasons for this are as follows: The size of particles of the $\mathrm{NCD} / \mathrm{W}$ in the particle formation process is bigger. In the growth process, therefore, the coalesced speed is faster and the gap of void is also narrower than that of the NCD/Ti.

In this paper, the formation and growth process of the NCD on the $\mathrm{W}$ and Ti layers are 
investigated which is very helpful to analyze many types of NCD deposition processes on various interlayers.

References :

[1] J. E. Field, Reports on Progress in Physics 75 (2012) 12.

[2] D. M. Gruen, Annu. Rev. Mater. Sci. 29 (1999) 211.

[3] B.-K. Na, C. H. Kang, J. Kor. Inst. Surf. Eng. 46 (2013) 68.

[4] H. Guo, Y. Qi, X. Li, J. Appl. Phys., 107 (2010) 033722.

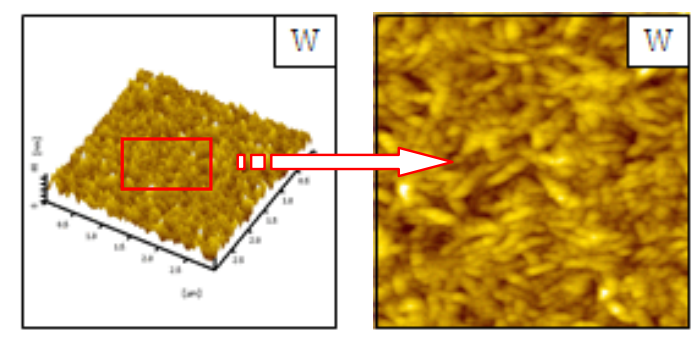

(a) $\mathrm{W}$

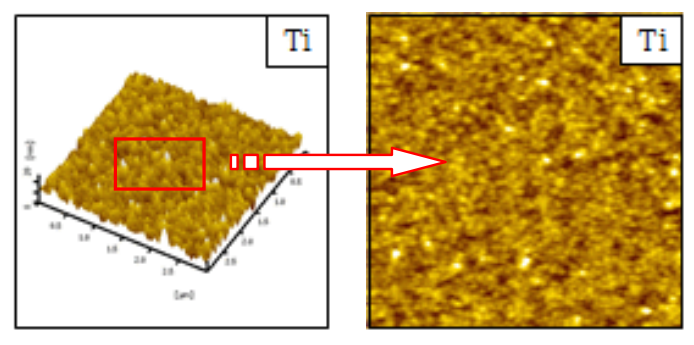

(b) $\mathrm{Ti}$

Fig. 1 AFM images of the $\mathrm{W}$ and the Ti interlayer.

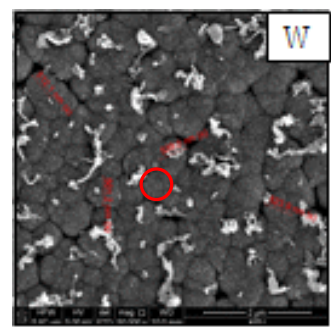

(a) NCD/W (at $0.5 \mathrm{~h})$

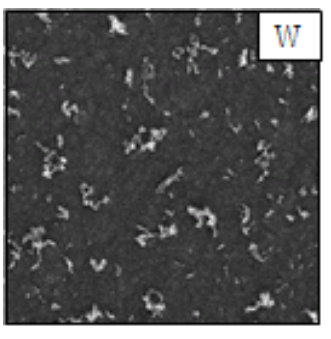

(b) NCD/W (at 2h)

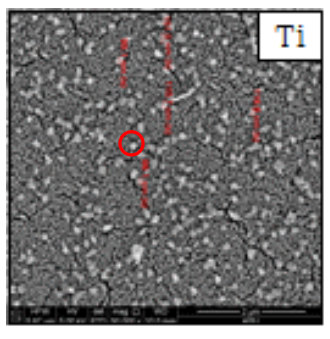

(c) NCD/Ti (at $0.5 \mathrm{~h}$ )

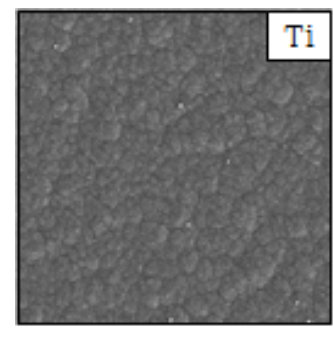

(d) NCD/Ti (at 2h)

Fig. 2 SEM images of the NCD/W and the NCD/Ti.

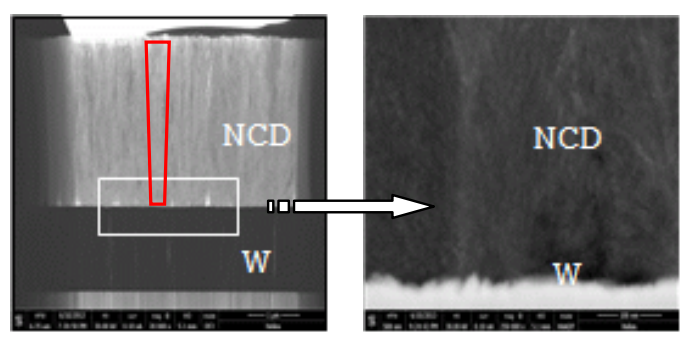

(a) NCD/W (at 2h)

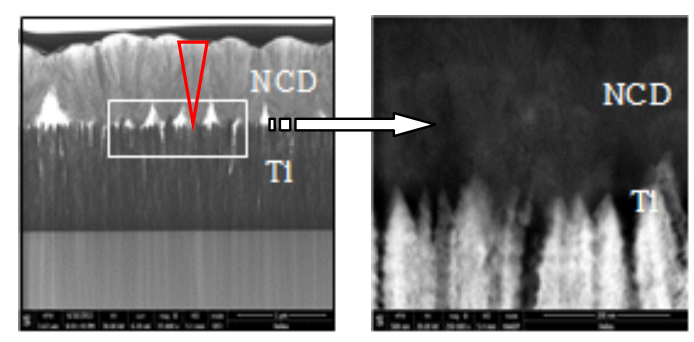

(b) NCD/Ti (at 2h)

Fig. 3 STEM images of the interface of the NCD/W and the NCD/Ti. 